\title{
Farnesol, tyrosol, and oxidative stress modulate the extracellular vesicle proteome and transcriptome in Candida albicans
}

\author{
Leitão Jr. NP1 ${ }^{1}$ Amorim MG ${ }^{2}$, Melo A, Mortara RA ${ }^{1}$, Sinigaglia-Coimbra R ${ }^{1}$, Tashima $\mathrm{AK}^{1}$, Nunes $\mathrm{D}^{2}$, \\ Silva $\mathrm{IT}^{2}$, Valieris $\mathrm{R}^{2}$, Dias-Neto $\mathrm{E}^{2}$ and Rosana Puccia
}

${ }^{1}$ Escola Paulista de Medicina-UNIFESP, São Paulo, Brazil; ${ }^{2}$ A. C. Camargo Cancer Center, São Paulo, Brazil

Background: EVs are closed structures, surrounded by a lipid bilayer membrane, which transport cellular components to the outside environment in all kinds of organisms. EVs are involved in cell signaling and immune modulation. Fungal EVs carry protein, lipid, polysaccharide, melanin, mRNA, s(small)RNA, and probably other molecules not yet described. Objetive: To characterize and compare the extracellular vesicles (EVs) produced by Candida albicans stimulated by farnesol, tyrosol, and oxidative stress.

Methods: We used concentration and differential centrifugation steps to isolate EVs from culture supernatants of $C$. albicans cultivated for one day under shaking at $37^{\circ} \mathrm{C}$ in Ham's F12 defined medium (pH 6.3) +1,5\% glucose (controls), in the presence of farnesol (50 $\mu \mathrm{M})$, tyrosol $(20 \mu \mathrm{M})$ or $25 \mathrm{mM} \mathrm{H}_{2} \mathrm{O}_{2}$. The stimuli did not alter viability or cell morphology. EVs were analyzed by nanoparticle-tracking for number and size, by microscopy for interaction with C. albicans and macrophages, and by liquid chromatography coupled with in-tandem mass spectrometry for quantitative proteomics. We used Bioanalyzer and RNA-seq (Proton platform, lon Torrent) for characterization of the mRNA and sRNA content in both EVs and C. albicans cells for each condition in biological triplicates.

Results not shown below: $C$. albicans EVs were up to $250 \mathrm{~nm}$ in diameter. Controls peaked at 40 and $92 \mathrm{~nm}$. Farnesol evoked about 8-fold increase in EV yields and a decrease in the mean EV size (one peak at $46 \mathrm{~nm}$ ). Tyrosol and oxidative stress had the opposite effect (lower yields and increased number of EVs around $100 \mathrm{~nm}$ ). C. albicans EVs interacted with and were uptaken by fungal cells, as shown by confocal microscopy. Bioanalyzer profiles of total RNA: EV RNA profiles were similar to control cells samples. EV/cell-farnesol sRNA peaked at 100 nt. EVs tended to be smaller in stressed EVs (100-150 nt) than in controls (100-250 nt). mRNA peaked at 400-700 nt. The stimuli affected EV mRNA at higher rates than cell mRNA. Uptake assays with PKH26-labelled fungal EVs showed that they were either bound to or internalized by $10 \%$ of cultured $C$. albicans cells. In our culture conditions about $10 \%$ of the cells were in the filamentous form.

General conclusions: Extracellular vesicles from C. albicans can signal fungal cells, decreasing their interaction with macrophages. Quorum sense molecules and oxidative stress can modulate the EV size, number, proteome, and transcriptome, thus suggesting that EVs might have a fundamental role in the fungal biology and virulence. Most importantly, comparison between EV and total C. albicans transcriptome suggested that the EV population does not reflect the cell mRNA population.

Financial Support: FAPESP, CNPq, CAPES

\section{Candida EV Proteome}
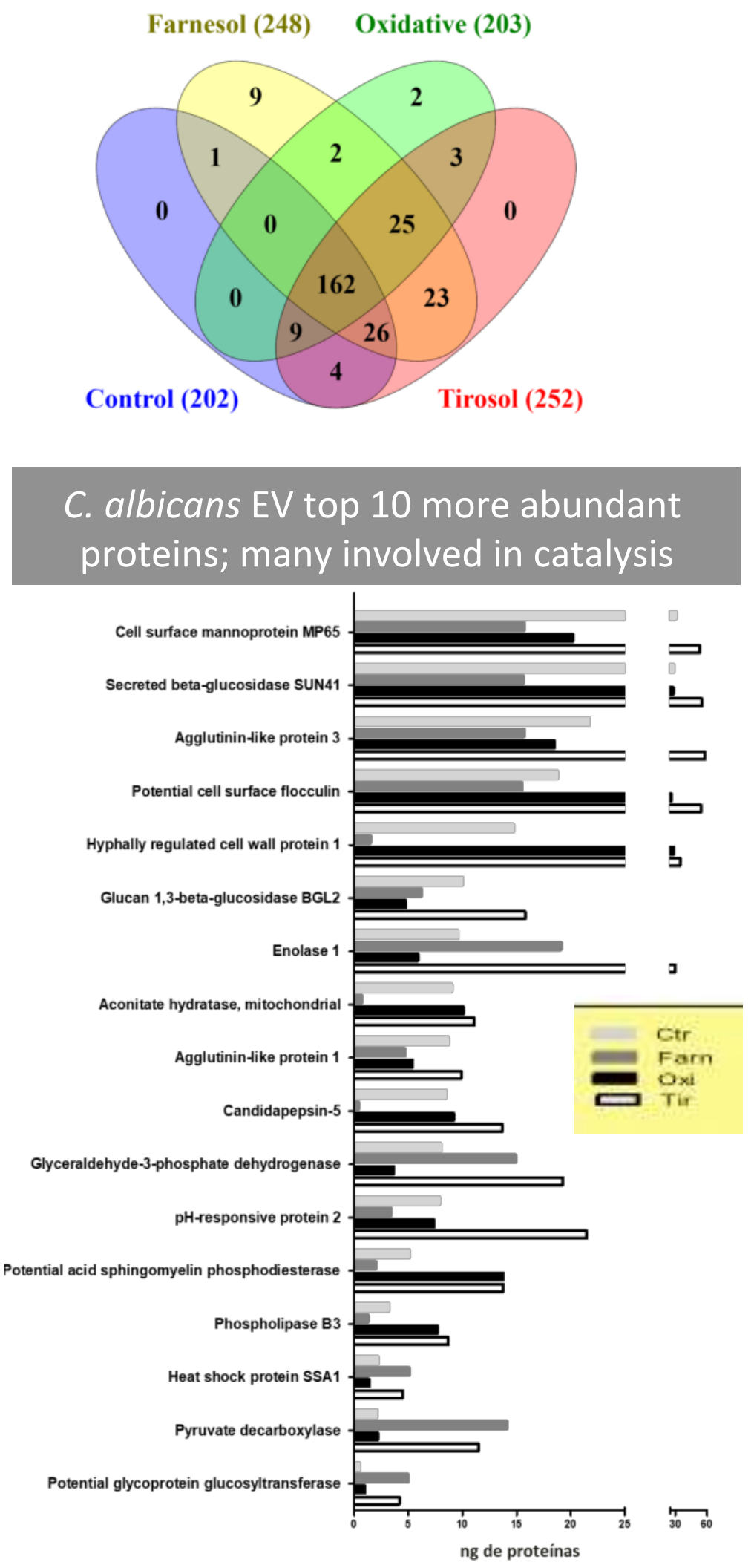

C. abicans EVs (stressed or not) negatively influenced fungal uptake and association index with macrophage Raw 264.7 (5:1 ratio)

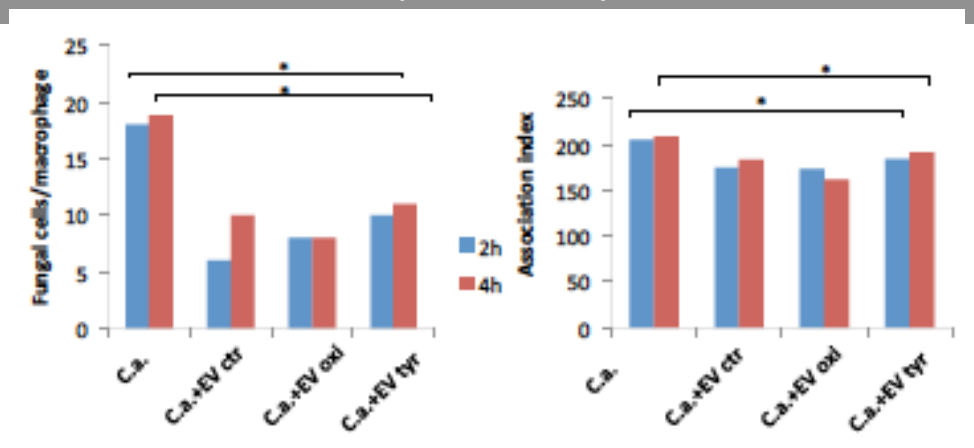

mRNA transcriptome (RNA-seq): Candida EVs $x$ cells (control, $\mathrm{H}_{2} \mathrm{O}_{2}$, farnesol, tyrosol)

Distance matrix shows high degree of concordance among samples in cell mRNA (1) and high degree of divergence between EV and cell samples (2,3); moderate divergence among EVs samples (4). In cells, divergence was mainly between mRNA from oxidative stress versus $\mathrm{crt}$ and tyrosol (a). That was partially reflected in EV mRNA (oxidative $\mathrm{x} \mathrm{crt,b)}$.

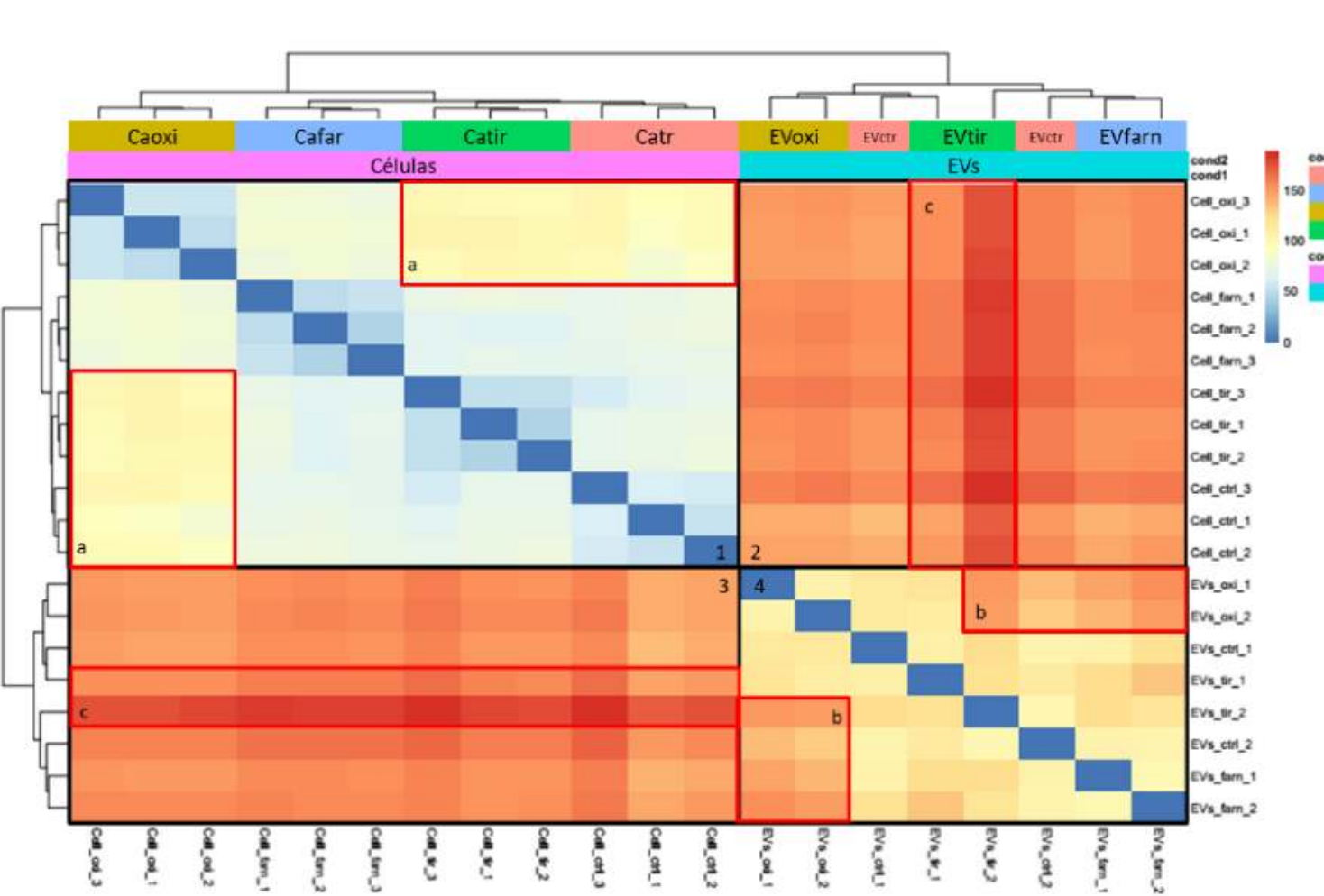

Overall results

842 total differentially represented mRNA in EV and cell samples

Number of modulated mRNA from EVs and cells ( $3 x$ difference, $p$ value $10^{13}$ ): EVs $x$ cells $=107$ ctr $x$ farnesol $=28$ $\operatorname{ctr} \times \mathrm{H}_{2} \mathrm{O}_{2}=119$ ctr $x$ tyrosol $=19$ farnesol $\mathrm{xH}_{2} \mathrm{O}_{2}=48$ farnesol $x$ tyrosol $=20$ $\mathrm{H}_{2} \mathrm{O}_{2} \times$ tyrosol $=140$

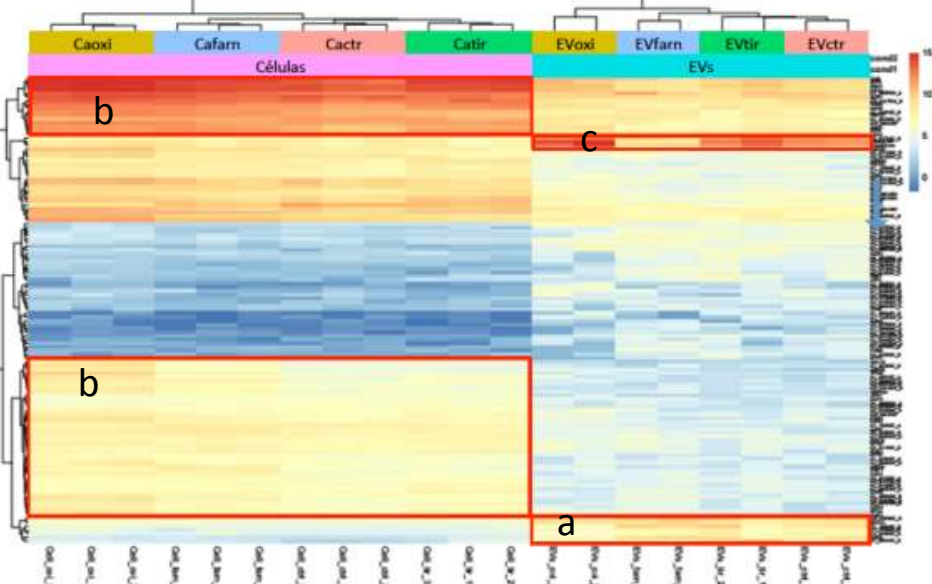

Among 107 differentially represented mRNA in EVs and cells, those in (a) include mRNA for Sec12 and Kip2 (biofilm and polarization); (b) 42 were $7 x$ richer in cells than in EVs, mostly with catalytic functions; (c) 10x more present in EVs tyrosol, $\mathrm{H}_{2} \mathrm{O}_{2}$, and ctr than in cell RNAs related to translation.

\section{Highlights from other heat map comparisons:}

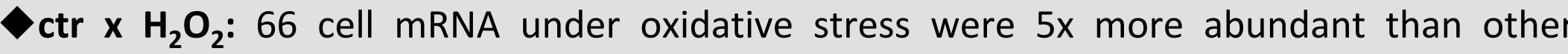
samples. Divergence with stressed $\mathrm{EV}-\mathrm{H}_{2} \mathrm{O}_{2}$ was higher. STL1 (for a hyphal chaperone) and TPO3 (for a yeast vacuolar protein) were 10x more abundant in the cells. Nine mRNA were more abundant in $\mathrm{EV}-\mathrm{H}_{2} \mathrm{O}_{2}$ (e.g.: RTA3, for a membrane stress protein and SEC9, for hyphal tSNARE related to exocytosis through the plasma membrane.

$\checkmark$ ctr $\mathbf{x}$ farnesol: the EV content partially reflects the cell mRNA, but EV samples were more distant. mRNA more abundant in EV-farnesol code for Hwp1, C6-02100W-A (stress), Mph1 (DNA replication).

$\checkmark$ ctr $\mathbf{x}$ tyrosol: the EV content partially reflects the cell mRNA. CUP9 (transcription), AOX2 (hyphal growth and biofilm), and YWP1 (for a cell wall protein; adhesion) were more abundant in cell mRNA. 